\title{
Systematic identification of intergenic long- noncoding RNAs in mouse retinas using full-length isoform sequencing
}

Ying Wan ${ }^{1,2}$, Xiaoyang Liu' ${ }^{1,2}$, Dongwang Zheng ${ }^{3}$, Yuying Wang ${ }^{1,2}$, Huan Chen ${ }^{4}$, Xiaofeng Zhao ${ }^{1,2}$, Guoqing Liang ${ }^{1}$, Dongliang $Y u^{1,2^{*}}$ (D) and Lin Gan ${ }^{5^{*}}$

\begin{abstract}
Background: A great mass of long noncoding RNAs (IncRNAs) have been identified in mouse genome and increasing evidences in the last decades have revealed their crucial roles in diverse biological processes. Nevertheless, the biological roles of IncRNAs in the mouse retina remains largely unknown due to the lack of a comprehensive annotation of IncRNAs expressed in the retina.

Results: In this study, we applied the long-reads sequencing strategy to unravel the transcriptomes of developing mouse retinas and identified a total of 940 intergenic IncRNAs (lincRNAs) in embryonic and neonatal retinas, including about 13\% of them were transcribed from unannotated gene loci. Subsequent analysis revealed that function of lincRNAs expressed in mouse retinas were closely related to the physiological roles of this tissue, including 90 lincRNAs that were differentially expressed after the functional loss of key regulators of retinal ganglion cell (RGC) differentiation. In situ hybridization results demonstrated the enrichment of three class IV POU-homeobox genes adjacent lincRNAs (linc-3a, linc-3b and linc-3c) in ganglion cell layer and indicated they were potentially RGCspecific.

Conclusions: In summary, this study systematically annotated the lincRNAs expressed in embryonic and neonatal mouse retinas and implied their crucial regulatory roles in retinal development such as RGC differentiation.
\end{abstract}

\section{Background}

Vertebrate retinas shares a highly organized and conserved structure, and is an excellent system to study the differentiation and development of neurons [1, 2]. Mouse (Mus musculus) retinas contain six major types of neurons and one type of glia that are involved in optical to visual signal transformation [3]. All retinal cells are generated from common retinal progenitor cells (RPCs) through conserved and concerted actions of a series of transcription factors, particularly components of homeodomain and bHLH families (e.g., Pax6, Six3 and Math5) [4-6]. Besides, increasing pieces of evidence in the last decade point to the crucial regulatory roles of

\footnotetext{
* Correspondence: yudl@hznu.edu.cn; lin_gan@urmc.rochester.edu ${ }^{1}$ College of Life and Environmental Sciences, Hangzhou Normal University, Hangzhou, China

${ }^{5}$ Department of Ophthalmology and Flaum Eye Institute, University of Rochester, Rochester, NY 14642, USA

Full list of author information is available at the end of the article
}

non-coding RNAs (ncRNAs) in retinal development and diseases, such as miRNAs (e.g., $m i R-124 a$, with its primary sequence termed Rncr 3 ) and long noncoding RNAs (lncRNAs) [7-9].

LncRNAs are defined as a class of RNAs greater than 200 nucleotides in length and without detectable coding potential [10]. LncRNAs are classified into several groups, including antisense, intronic and intergenic (lincRNAs) according to the relationship between lncRNAs and their associated protein-coding genes [11]. It is now widely accepted that lncRNAs play pivotal regulatory roles in diverse biological processes, such as imprinting, cell cycle regulation, cell differentiation, and other developmental processes $[12,13]$. During mouse retinogenesis, for instance, Tug1 is necessary for photoreceptor formation [14], Miat (also known as Rncr2 or Gomafu) and Six3OS are involved in regulating the retinal cell fate specification [15, 16], Vax2os is involved in the control of 
cell cycle progression of photoreceptor progenitor cells in the ventral retina [17], and Malat1 is functional associated with retinal microvascular dysfunction and neurodegeneration [18, 19]. Moreover, a requirement for a complex comprising of miRNA (miR-183/96/182), lncRNA (Rncr4) and protein (RNA helicase $\operatorname{Ddx} 3 \mathrm{x})$ in the organization of retinal architecture is also revealed [20]. In a more recent study, 119 lncRNAs were identified with their potential roles in regulation of photoreceptor cell differentiation [21]. All these facts revealed the importance of lncRNAs during retina formation and development, however, in view of the huge number of lncRNAs in mouse genome [22, 23], our understanding about the biological roles of lncRNAs in the differentiation and development of mouse retinal neurons remains limited, and a systematic annotation of lncRNAs expressed in the retina is still lacking.

In the present work, we carried out a long-reads sequencing of transcripts expressed in mouse retinas at developmental stages encompassing the generation of all retinal cell types and performed a genome-wide analysis of the intergenic lncRNAs (lincRNAs). About one thousand lincRNAs are identified and the subsequent function and expression analysis argue for future exploration of the roles of lncRNAs in regulating retinal development.

\section{Methods}

\section{Mice}

Mouse experiments in this study were performed according to protocols approved by the Ethics Committee for Experimental Animals at Hangzhou Normal University (permit number: 2018069). C57BL/6 J mice were used in this study. All the used mice were provided by the experimental animal center of Hangzhou Normal University. Mice, no more than ten, were euthanasized by carbon dioxide ( $20 \%$ chamber volume per minute, at least $10 \mathrm{~min}$ ) in individually ventilated cages, followed by cervical dislocation. Retinas were dissected from embryos at embryonic days of E12.5, E14.5, E16.5 and E18.5, and from postnatal mice at P0, P3, P5, P7, P14, P21 and P28. At least three individuals were required for each developmental stage. Separation of retinas was performed according to the previously described protocol [24]. Curved scissor was used to push the eye out of its socket after killed the mouse by euthanasia. The optic nerve was cut and the eyes were removed into petri dish containing ice cold diethyl pyrocarbonate treated PBS (0.1\%; Sigma, cat no: D5758). Subsequently, we used the forceps to poke a hole on the cornea and gently tear the hole to detach the retina away from the retinal pigmented epithelium. Then, the sclera, iris, lens and any other remaining structures were carefully removed until the retina was completely isolated. The isolated retinas were put into $1.5 \mathrm{ml}$ RNase-free microcentrifuge tubes and stored in $-80^{\circ} \mathrm{C}$ for further experiments.

\section{RNA preparation and sequencing}

Retinas from embryonic and postnatal individual were respectively pooled prior to RNA isolation. The total RNAs were isolated using the RNeasy kits (Qiagen, Valencia, CA) in accordance with the manufacturer's protocol, respectively. RNA degradation and contamination were monitored on $1 \%$ agarose gels. The purity of RNA samples was checked using the NanoPhotometer spectrophotometer (IMPLEN, CA, USA). RNA concentration was measured using Qubit RNA Assay Kit in Qubit 2.0 Flurometer (Life Technologies, CA, USA). The integrity of RNA was assessed using the RNA Nano 6000 Assay Kit of the Agilent Bioanalyzer 2100 system (Agilent Technologies, CA, USA). $2 \mu \mathrm{g}$ qualified RNAs (OD260/ 280: $2.0-2.2$, OD260/230: $1.8-2.1$ and $\mathrm{RIN} \geq 9.0$ ) from each of the pooled embryonic and postnatal mouse retinas were mixed.

A total of $4 \mu \mathrm{g}$ mixed RNA was used for full-length transcript sequencing with Pacbio Sequel system (Pacific Biosciences, CA, USA) according to the manufacturer's instructions. The Iso-Seq library was prepared according to the Isoform Sequencing (Iso-Seq) protocol using the Clontech SMARTer PCR cDNA Synthesis Kit and the BluePippin Size Selection System as described by Pacific Biosciences (PN 100-092-800-03). Another $1.5 \mu \mathrm{g}$ mixed RNA was used for short reads sequencing on HiSeq X Ten platform (Illumina, CA, USA), with the library constructed using NEBNext UltraTM RNA Library Prep Kit for Illumina (NEB, USA) following manufacturer's recommendations.

\section{Iso-seq data processing and error correction}

Raw data produced by Pacbio was processed by the SMRT Link package (v5.0) (http://www.pacb.com/support/software-downloads/). Reads with a length $<200$ nt or with the ReadScore $<0.75$ were filtered out before generating the circular consensus sequence (CSS) $($ minPasses $=1, \quad$ minPredictedAccuracy $=0.8) . \quad$ FLNC (full-length non chimera) and NFL (non full-length) reads were then classified by checking the signals of $5^{\prime}$ - and $3^{\prime}$ - primers, as well as the poly-A tail. ICE algorithm was used to cluster the FLNC reads to generate the consensus sequences, which were then polished using the NFL reads. LoRDEC (Version 0.6) was used to correct these consensus sequences with the Hiseq generated short reads [25], which were priory filtered to remove the reads with the ambiguous bases $>10 \%$ or with the low quality bases (Q-value $<20)>50 \%$ in length. 


\section{Identification of IncRNAs}

The reference mouse genome was downloaded from GENCODE (mm10, www.gencodegenes.org). The corrected consensus sequences were mapped to the mouse genome by using GMAP (Version 2017-01-14) (--expand-offsets 1 -B 5 -K 50000 -f samse - n 0 -t 4) [26]. Redundant sequences were removed by using Cupcake ToFU package (https://github.com/Magdoll/ cDNA_Cupcake) and CD-HIT package (CD-HIT-EST, -c 0.95 -aS 0.95) [27]. The collapsed Pacbio transcripts were then mapped back to mouse genome again using STAR [28].

CPAT (v1.2.4) was used to remove the protein-coding transcripts first, i.e., any transcript with the coding probability $>0.44$ was considered as a coding sequence [29]. Thereafter, open reading frames (ORFs) of the retained transcripts were detected by TransDecoder (v5.2.0). Their deduced peptides ( $>100$ amino acids) were compared to the known mouse proteins using BLASTP [30], which were meanwhile scanned for potential domains by using HMMER (Version 3.2.1, --cut_ga) (hmmer.org) and PFAM domain profiles [31]. Transcripts were filtered out if their encoded peptides showed domain architectures or shared $>85 \%$ identity to known proteins with the alignment region longer than 50 amino acids. Subsequently, BLASTN was used to align the retained Pacbio transcripts to all the mRNA of characterized mouse protein-coding genes. Transcripts were regarded as partial protein-coding transcripts if $>95 \%$ identity was observed across the aligned region that covered $>50 \%$ of the query transcripts in length.

In addition, based on the bam file from STAR alignment, MatchAnnot (https://github.com/TomSkelly/ MatchAnnot) was used to compare the structures between the Pacbio transcripts and the known genes. Pacbio transcripts were removed from the collection of lncRNA candidate if they were perfectly matched the protein-coding or institute RNA genes in structure (score 5). Moreover, the Pacbio transcripts exactly matched one or more introns of protein-coding genes were also filtered out. The lincRNAs were then picked out by comparing the genomic location to known genes by cuffcompare (Cufflinks package, v2.2.1) [32]. Cuffcompare was also used to compare the locations and structures of lincRNAs identified in this work to previous annotation [21, 33, 34]. Notably, for IncRNAs deposited in NONCODE, their expression in particular tissues were accepted only when the FPKM (Fragments Per Kilobase of transcript per Million fragments mapped) > 0.01 . Unless otherwise specified, default parameters were selected for the bioinformatics tools mentioned above.

\section{Short reads mapping and abundance calculation}

Short reads generated from HiSeq were mapped to the all the mappable full-length transcripts acquired in this work, as well as all the protein-coding transcripts that annotated in GENCODE, by using BOWTIE (version 1.1.2) with no more than two mismatchs allowed [35]. Abundance of lncRNAs was measured by RPKM (Reads Per Kilobase per Million mapped reads) that was calculated by in-house developed perl scripts. Significance of abundance variation between different groups was calculated using Student's t-test function of R package (version 3.5.0).

\section{Function analysis of lincRNA nearby genes}

According to the genome annotation information from GENCODE release, protein-coding genes within $100 \mathrm{~kb}$ of lincRNA loci were collected as potential cis-targets $[36,37]$. Functional enrichment analysis was performed through the Enrichr webserver [38], with the redundant GO terms were removed by Revigo (allowed similarity 0.7) [39]. Significance of the domain enrichment was assessed by the $p$-value generated from Fisher exact test (cutoff 0.05).

\section{Identification of differentially expressed lincRNAs}

RNA-seq data of retinas from wild type, Math5-null, Isl1-null and Pou4f2-null mice at E14.5 were downloaded from NCBI Sequence Read Archive (SRA), which were deposited under the accession number SRP037539 [40]. Expression level of identified lincRNAs were calculated with the same strategy as described in 'Short reads mapping and abundance calculation'. Differentially expressed isoforms were identified by using $\mathrm{R}$ package DEGSeq [41], with the cutoff fold change $\geq 2$ and p-value $\leq 0.05$.

\section{Reverse transcription PCR}

Reverse transcription (RT)-PCR was used to validate the transcription of novel lincRNAs. Total RNA was extracted from pooled mouse retinas the same way as described in the RNA preparation for sequencing. DNA-free RNA was reverse transcribed to cDNAs using PrimescriptTM RT reagent Kit (Takara, Code No. RR047A). All PCRs were performed in $20 \mu \mathrm{l}$ volume containing $4 \mu \mathrm{l}$ 5X PrimeSTAR buffer, $1.6 \mu \mathrm{l}$ dNTP mixture $(2.5 \mathrm{mM}), 0.5 \mu \mathrm{l}$ primers $(10 \mu \mathrm{mol} / \mathrm{L}), 0.2 \mu \mathrm{l} \mathrm{DNA}$ polymerase $(2.5 \mathrm{U} / \mu \mathrm{l})$ (R010A, Takara), $1 \mu \mathrm{l}$ cDNA and $12.2 \mu \mathrm{l}$ nuclease-free water. The PCR products were sequenced by ABI 3730XL DNA Analyzer (ABI/Life Technologies, USA) and aligned to the mouse genome using BLAT through the UCSC Genome Browser web service (genome.ucsc.edu).

\section{Fluorescence in situ hybridization (FISH)}

The lncRNA probe templates, with the T3 and T7 adaptors, were firstly synthesized and inserted in pUC57 vector (Genewiz, Suzhou, China) and then amplified with the M13 primers by PrimeSTAR HS DNA Polymerase 
(Takara, Code No. R010A) (see Additional file 8: File S1 for probe sequences). Sense and antisense of probes were transcribed with T7 and T3 RNA polymerases, respectively. DIG RNA labeling mix (Roche, 1,277,073) was used to label the probes. Probes of Pou4f1, Pou4f2 and Pou4f3 were used as the positive control, which were generated according to the method described previously [42].

Retinas from mice at E15.5 were used for detection of gene expression. The embryos at E15.5 were fixed in 4\% paraformaldehyde in PBS at $4{ }^{\circ} \mathrm{C}$ for $6 \mathrm{~h}$ and dehydrated in $30 \%$ sucrose in PBS at $4{ }^{\circ} \mathrm{C}$. After dehydration, the embryos were embedded in OCT (ThermoFisher Thermo NEG50, cat. no. 6506) at $-80^{\circ} \mathrm{C}$. FISH on sectioned tissues were carried out according to the previously described method [43]. Probes were detected by using Anti-digoxigenin-HRP (1:1000) (Perkin Elmer, cat. no. NEF832001EA) and Tyramide Signal Amplification system (Perkin Elmer, TSA Plus, cat. no. NEL744001KT) according to the manufacturer's recommendation.

\section{Results}

Iso-seq and error correction

Sequencing of pooled mouse retinal RNAs generated a total of 5,084,169 subreads from six SMAT cells of combined SMRTbell libraries, which formed 372,267 higher quality circular consensus sequences (CCSs). 295,179 FLNC sequences, i.e., CCSs containing the $5^{\prime}$ - and $3^{\prime}$ primers and polyA tails, were subsequently clustered and polished by using NFL sequences that yielded a total of 172,333 consensus sequences. LoRDEC was used to improve the quality of the Iso-seq acquired consensus sequences, using about $195 \mathrm{M}$ high-quality short reads generated by RNA-seq of the pooled retinas. After error correction, the total nucleotide of the 172,333 consensus sequences was increased from 501.8 to $505.1 \mathrm{M} \mathrm{bp}$, whilst the average length and N50 were also slightly increased (Additional file 1: Table S1).

\section{Identification of IncRNA genes}

Based on the mapping of the corrected consensus sequences to the mouse genome by using GMAP, a total of 120,297 transcripts ( $\geq 200 \mathrm{nt}$ ) were reconstructed using the Cupcake ToFU package. CD-HIT-EST was used to filter out the transcripts varied only in the $5^{\prime}$ or $3^{\prime}$-end and generated 71,251 collapsed transcripts. 97.5\% of these transcripts $(69,495)$ were mapped to the mouse genome with STAR (error rate per base $0.01 \%$ ) and were selected for further identification of noncoding transcripts.

Identification of lncRNAs in this work was performed according the strategy described in Fig. 1. First, coding potential of the candidates was calculated by CPAT, which filtered out 21,911 transcripts that showed a coding probability $>0.44$. Secondly, a total of 56,126 long ORFs (with the deduced peptides $>100$ amino acids) were detected in 29,027 of the remaining transcripts, including 13,523 ORFs showed significant domain features and 16,251 shared high similarity to known mouse proteins, respectively. Transcripts contained any of these ORFs were filtered out, resulting in a collection of 33,060 transcripts. Primary nucleotide sequence based homology search (BLASTN) revealed that 3306 transcripts were mapped to the characterized $5^{\prime}$ or 3'- untranslated regions (UTRs), which were therefore considered as fragments of protein-coding transcripts.

Moreover, exon-intron structures of the retained 29, 754 transcripts were compared to annotated mouse genes with MatchAnnot. Transcripts were filtered out if their structures perfectly matched the known proteincoding / institute RNA genes (e.g., microRNAs, snoRNAs and snRNAs) or they exactly shared one or more introns with protein-coding genes, which eventually resulted in 27,867 candidate lncRNAs, including a total of 5404 ones with multiple exons. At least two short reads from the RNA sequencing of pooled retina sample were mapped to $93.4 \%$ of these candidates, i.e., $96.4 \%$ (5209/ $5404)$ and $93 \%(20,866 / 22,463)$ for multiple-exon and single-exon encoded candidates, respectively, indicating they were generated at low risk of DNA contamination.

Notably, transcripts derived from Iso-seq have not been subsequently mapped to specific DNA strands in this work, thus it is difficult to accurately distinguish the antisense/intronic lncRNAs from the isoforms of protein-coding genes in many cases, although a number of candidate lncRNAs could be classified into antisense/ intronic lncRNAs with high confidence based on the similarity of primary sequences and structures. By checking the chromosomal locations of the identified 5404 multiple-exon encoded lncRNA candidates, 940 transcripts that non-overlapped with any known proteincoding genes were selected for subsequent analysis (Additional file 9: File S2). Exon-intron structures of ten randomly selected lincRNAs, at least one for each, were validated by RT-PCR and products analysis (Additional file 2: Table S2, Additional file 6: Figure S1).

\section{General features of retinal lincRNAs}

The identified 940 multiple-exon encoded retinal lincRNAs were transcribed from 690 putative gene loci and their sizes ranged from 202 to $14,455 \mathrm{nt}$. Their average length of about $2600 \mathrm{nt}$ was significantly longer than the known lincRNAs (Fig. 2a). Despite of the variations in length, the number of exons per lincRNA was similar between lincRNAs identified in this work and those deposited in GENCODE (vM17), i.e., about half of the lincRNAs contained two exons and $>90 \%$ lincRNAs 


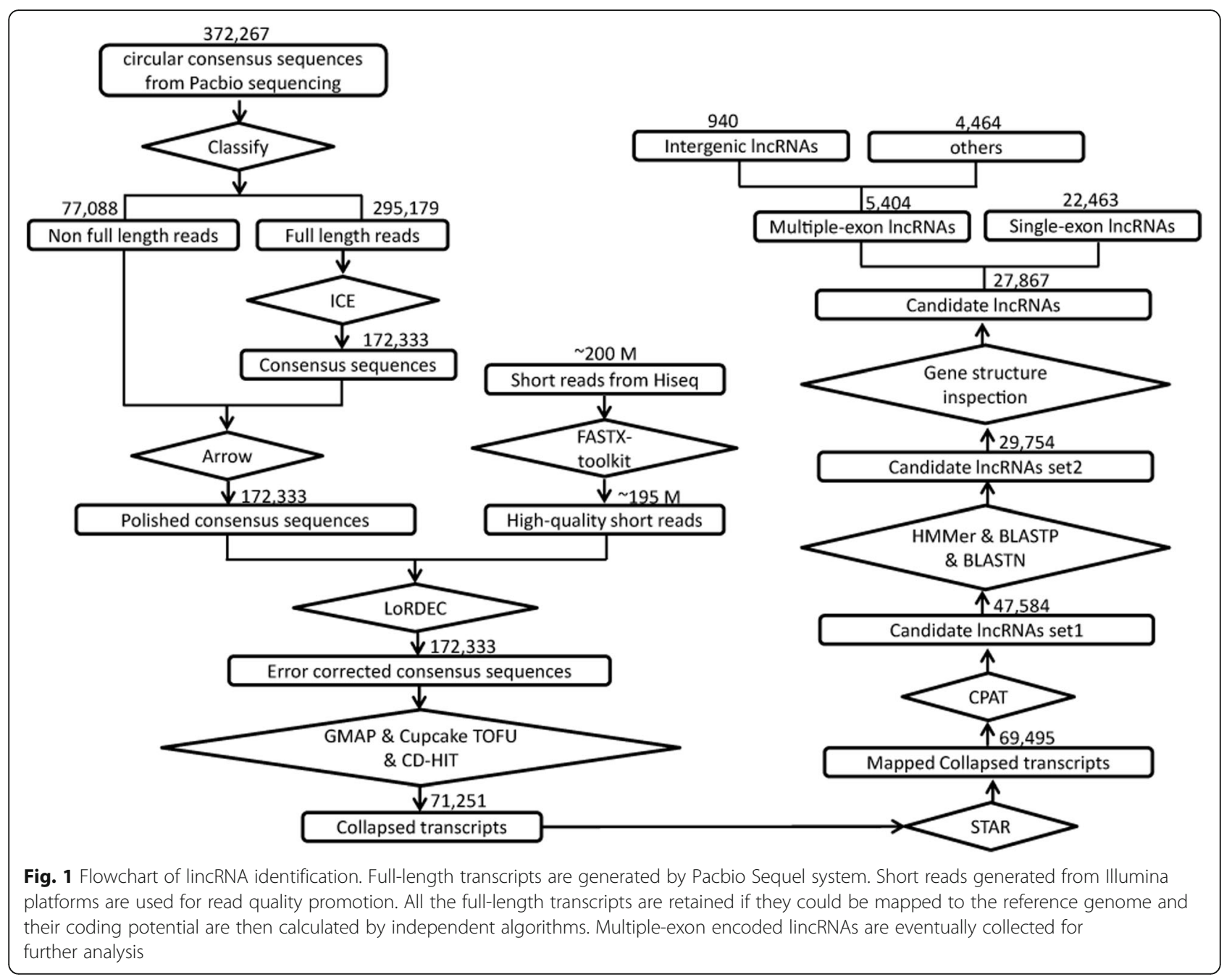

contained fewer than five exons (Fig. 2b). Retinal lincRNAs containing the largest number of exons were transcribed from the same locus, i.e., a total of 14 lincRNAs were transcribed from a $265 \mathrm{~kb}$ genomic region (chr7:61, 944,240-62,209,515), including eight of them had more than ten exons.

By comparing the 940 retinal lincRNAs to the GENCODE annotation, we found that about half of them (434/940) were transcribed from novel gene loci. The rest were overlapping with known lincRNAs, including 56 showed exactly the same structures to their counterparts. Taking the previously characterized lincRNA genes Miat and Tug1 for example, we also observed several novel isoforms in addition to the known transcripts (Fig. 3).

The expression of mouse lncRNAs has been investigated in several tissues like brain, liver, heart, and testes, and the expression profiles could be retrieved from NONCODE database and the work of Zhao et al [33, 34]. Comparative analysis revealed that a total of 122 lincRNAs (13\%), transcribed from 112 potential gene loci, were only found in mouse retinas. The other lincRNAs either shared the same structure $(8.3 \%)$ or overlapped with the known lncRNAs (78.7\%). In addition, we also checked the expression of those 940 lincRNAs in developmental photoreceptors (P2-28) [21]. By comparing the chromosomal location to the assembled transcripts in photoreceptors, we found that 548 (58.3\%) of the identified lincRNAs were transcribed from the transcription-active loci in photoreceptors, including 75 lincRNAs that shared the same structures with the transcripts expressed in photoreceptors (Additional file 3: Table S3). In contrast, the other 392 (41.7\%) lincRNAs were not overlapping with any gene expressed in photoreceptors, indicating they might be expressed in photoreceptors at the stages other than P2-P28 or in the other cell types in retina.

\section{Abundance of lincRNAs}

Short reads generated from sequencing of the pooled retinal RNAs were used to calculate the relative abundance of the lincRNAs. About $71.4 \%$ of the short reads 
A

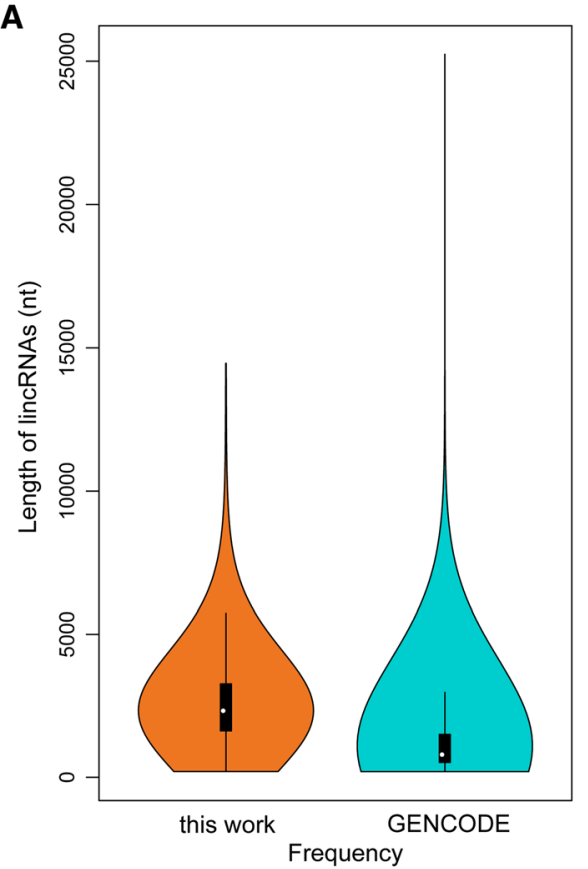

B

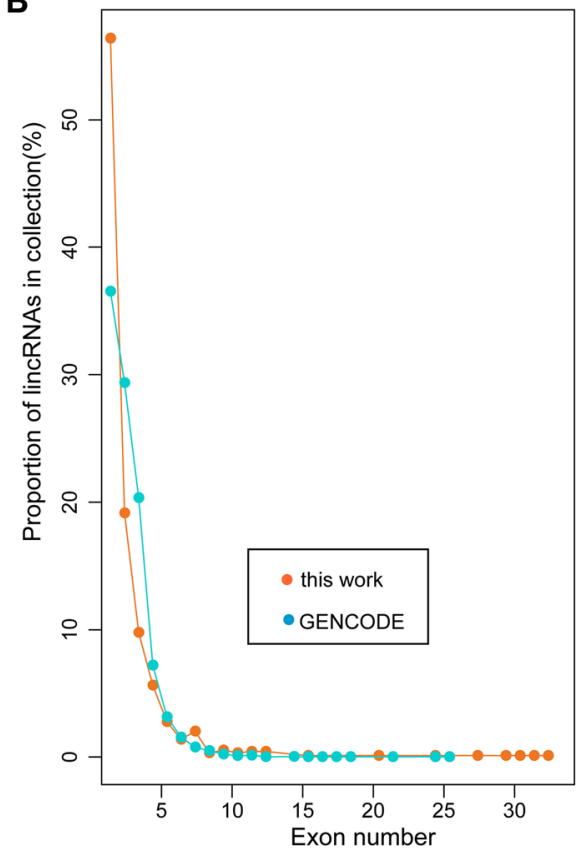

Fig. 2 Genomic features of retinal lincRNAs. (a) Length distribution of lincRNAs identified in the mouse retina and annotated by GENCODE. (b) Statistics of exon numbers in lincRNAs identified in the mouse retina and previous annotation

could be aligned to $98 \%$ of the clustered full-length transcripts $(69,495$ in total). Consistent with the known knowledge that the noncoding RNA genes are usually expressed at a low level, the abundance of the 940 lincRNAs were significantly lower than those with coding potential, including only about $25 \%$ of them with the RPKM > 1 (Fig. 4a). Moreover, the 122 potential retinaspecific lincRNAs were expressed at a remarkable lower level than multiple-tissue expressed lincRNAs (two folds in RPKM, $p<0.001$ ) (Fig. 4b).

In contrary to the low expression level of most lincRNAs, several lincRNAs (non-repeat elements) were abundant in the investigated sample with the RPKM > 10 (Table 1). Interestingly, about half of these abundant lincRNAs were transcribed from Miat, Malat1, and Rncr3, which were involved in different processes of retinal development as mentioned above. Besides, we noticed that $C d k n 1 b$, neighboring to lincRNA gene Lockd at $\sim 3.5 \mathrm{~kb}$ distance, is involved in controlling the timing of cell cycle exit of retinal progenitors and is required to maintain the quiescence of bipolar cells, Müller cells, and cones [44]. Given that cis-regulation is a common mechanism of lincRNAs acting [45], the role of Lockd in retinal development is worthy of further exploration. Moreover, according to the expression profiles from NONCODE, these abundant lincRNA genes were also active in other tissues, such as liver, thymus, lung, spleen, heart or hippocampus. Notably, a half of their transcripts identified in the retina were novel that further study is required to confirm their retina-specific expression.

\section{Function analysis of lincRNA neighboring genes}

As high-throughput methodology directly towards the function of lincRNAs remains unavailable, biological roles of IncRNAs are usually inferred from the nearby protein-coding genes (cis-targets). Herein, 482 proteincoding genes nearby the identified lincRNAs were identified (within $100 \mathrm{~kb}$ ). Results from homology search (BLASTP, $\geq 85 \%$ identity) and short reads mapping ( $\geq 2$ reads) revealed that almost all of these 482 genes were expressed in developmental retinas (465/482). These potential cis-targets included several transcription factors that play crucial roles in retinal development, with the largest family as homeobox genes, such as Six6, Lhx1, Onecut1, Six3, Otx2, Pax6, Pou4f1, Pou4f2 and Pou4f3. Interestingly, in this study, no lincRNA was identified nearby bHLH genes that are characterized as important regulators of retinal cell differentiation, such as Math5, NeuroD, Mash1, and Hes1. GO enrichment analysis revealed that these lincRNA neighboring protein-coding genes were largely associated with neuronal differentiation and development, e.g., the most significantly enriched biological process GO categories were axonogenesis (GO:0007409) and negative regulation of neuron differentiation (GO:0045665) (Fig. 5). Likewise, for those potentially retina specific lincRNAs, their nearby protein-coding genes were enriched in the same or 


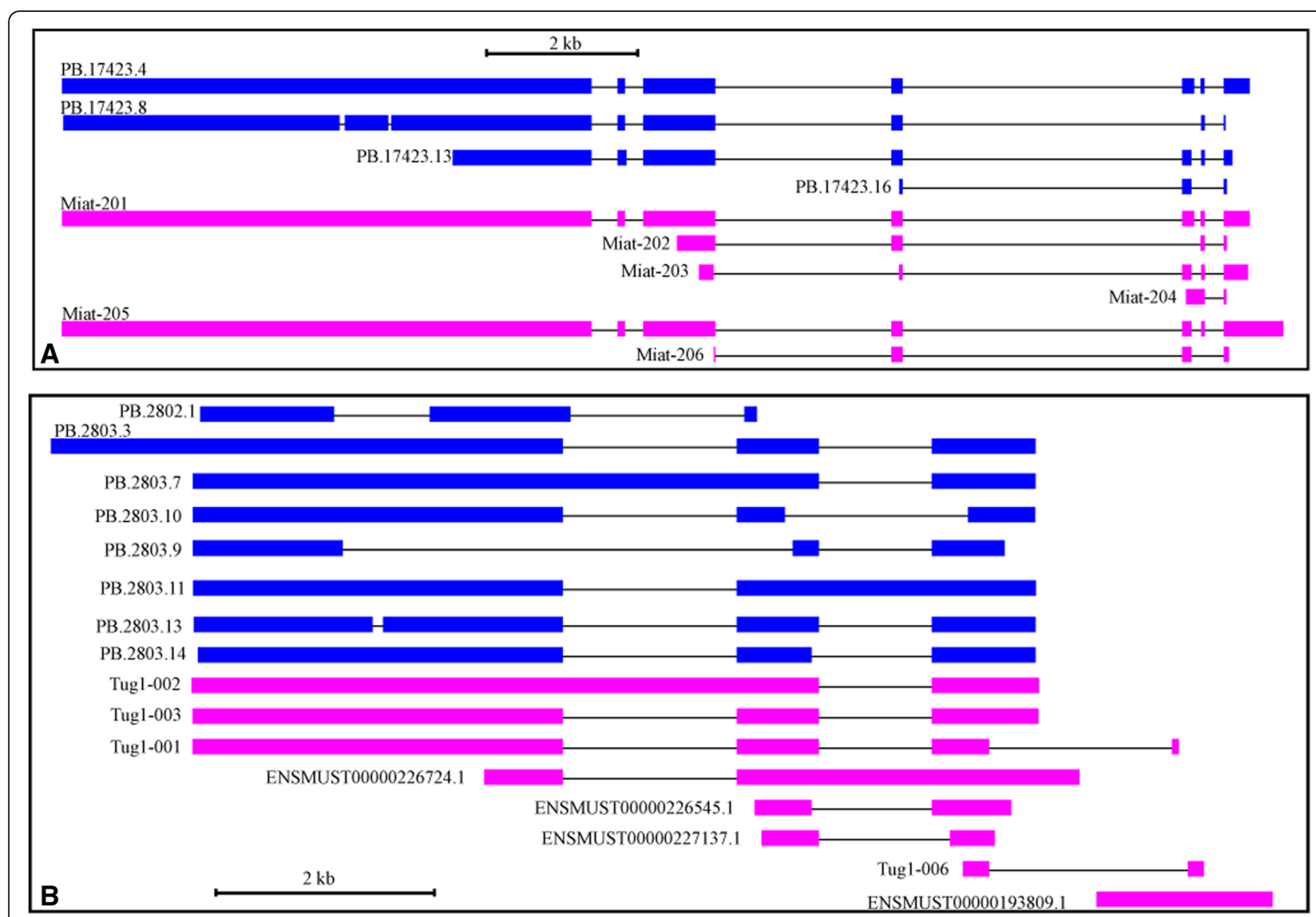

Fig. 3 Structures of lincRNA genes Miat and Tug1. The blue and pink blocks indicate the exons of transcripts generated from Iso-seq (PB.x.X) and annotated in GENCODE (VM17), respectively. The lines between blocks represent introns. Both the exons and introns are drawn to scale. (a) Miat locus; (b)Tug1 locus

related processes, such as axonogenesis, visual perception (GO:0007601) and sensory perception of light stimulus (GO:0050953) (Additional file 4: Table S4).

\section{Identification of lincRNAs associated with RGC regulators} MATH5, ISL1 and POU4F2 are key regulatory factors of RGCs specification and differentiation. MATH5 is required for establishing RGC competence within a subset of RPCs [46, 47]. Likewise, functional loss of Isl1 and Pou4f2 leads to the apoptosis of most RGCs before birth [48-51]. In addition, many other transcription factors involved in the regulation of RGC differentiation have been identified as well, such as SOX4 and SOX11 [52]. Nevertheless, little is known about the biological roles of lncRNAs during RGC differentiation and development.

In order to investigate lincRNAs that may be involved in the regulation of RGC differentiation, we analyzed the RNA-seq data of mice null for Math5, Isl1 or Pou4f2, and found that a total of 90 lincRNAs, transcribed from 85 non-overlapped genomic regions, were differentially expressed (Table 2). Functional analysis of their nearby protein-coding genes revealed enrichment of several biological processes, such as negative regulation of neuron differentiation, axonogenesis and embryonic camera-type eye morphogenesis (Additional file 5: Table S5).

In Math5-null retinas, 22 and 31 lincRNAs were upand down-regulated, respectively. As the targeted deletion of Math5 leads to the agenesis of almost all RGCs, it is possible that the down-regulation of some lincRNAs were caused by the loss of RGCs. Expression of two thirds of these down-regulated lincRNAs (21/31) were not detected in photoreceptors, including three of them, i.e., PB.7356.2 (linc-3a), PB.21631.1 (linc-3b), and PB.10720.6 (linc-3b), were resided next to the RGC specific expressed genes Pou4f1, Pou 4 f2 and Pou4f3 (within $10 \mathrm{~kb}$ ), respectively [53]. Different versions of mouse genome annotation have specified the lncRNA gene D130079A08Rik / RP23-67A17.1 that overlapped linc-3a, as well as Gm31501 / Gm31438 that overlapped linc-3c (Additional file 7: Figure S2), whereas $l i n c-3 b$ was identified in this work. Interestingly, by using FISH detection in retinal sections, our further work demonstrated that expression of linc-3a, linc-3b and linc-3b were enriched 

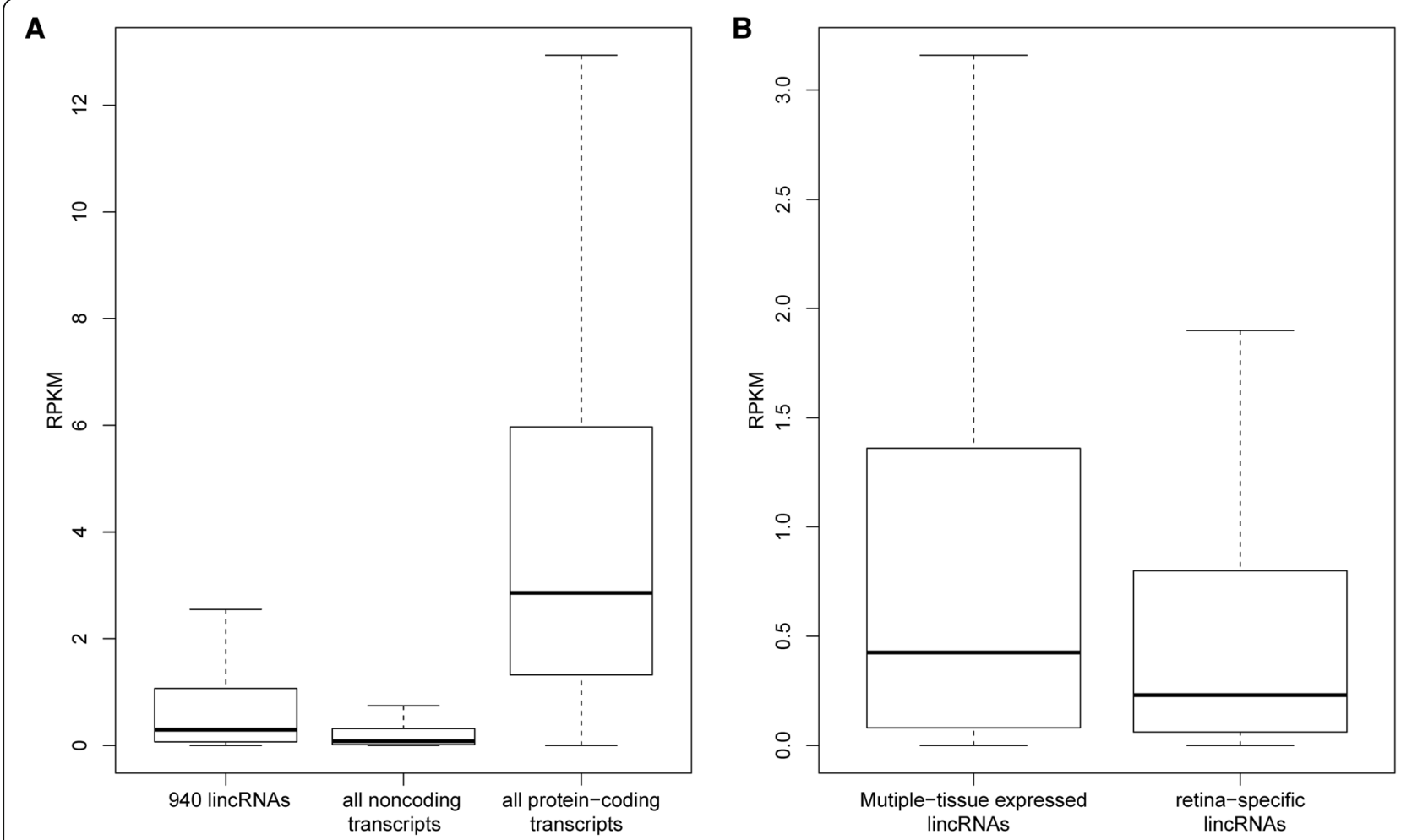

Fig. 4 Abundance of the lincRNAs expressed in mouse retina. (a) Comparison of the abundance between coding/noncoding transcripts and lincRNAs. (b) Comparison of abundance between retina-specific and multiple-tissue expressed lincRNAs. Transcript abundance is calculated by RPKM

Table 1 Top 20 abundant lincRNAs expressed in the mouse retina

\begin{tabular}{|c|c|c|c|}
\hline Transcripts & Host IncRNA gene & Relative abundance (RPKM) & Compare to GENCODE annotation \\
\hline PB.20890.2 & $\mathrm{H} 19$ & 83.81 & known \\
\hline PB.17423.4 & Miat & 72.23 & known \\
\hline PB.11213.21 & Malat1 & 70.83 & novel isoform \\
\hline PB.17423.8 & Miat & 65.29 & novel isoform \\
\hline PB.17423.13 & Miat & 40.82 & known \\
\hline PB.7082.15 & Rncr3 & 21.84 & novel isoform \\
\hline PB.7083.1 & Rncr3 & 20.3 & novel isoform \\
\hline PB.13759.2 & Mir124-2hg & 20.08 & novel isoform \\
\hline PB.19312.10 & Lockd & 19.78 & known \\
\hline PB.5352.9 & Rian & 19.32 & known \\
\hline PB.6163.26 & C130071C03Rik & 18.9 & known \\
\hline PB.7082.1 & Rncr3 & 18.38 & known \\
\hline PB.18345.2 & 1810058|24Rik & 18.02 & known \\
\hline PB.12880.18 & 1700020l14Rik & 16.48 & known \\
\hline PB.7082.17 & Rncr3 & 16.43 & novel isoform \\
\hline PB.7082.5 & Rncr3 & 14.23 & novel isoform \\
\hline PB.12880.30 & 1700020l14Rik & 13.84 & novel isoform \\
\hline PB.13343.15 & 2900097C17Rik & 12.94 & novel isoform \\
\hline PB.1224.18 & Gas5 & 12.92 & novel isoform \\
\hline PB.13344.4 & 2900097C17Rik & 12.74 & novel isoform \\
\hline
\end{tabular}




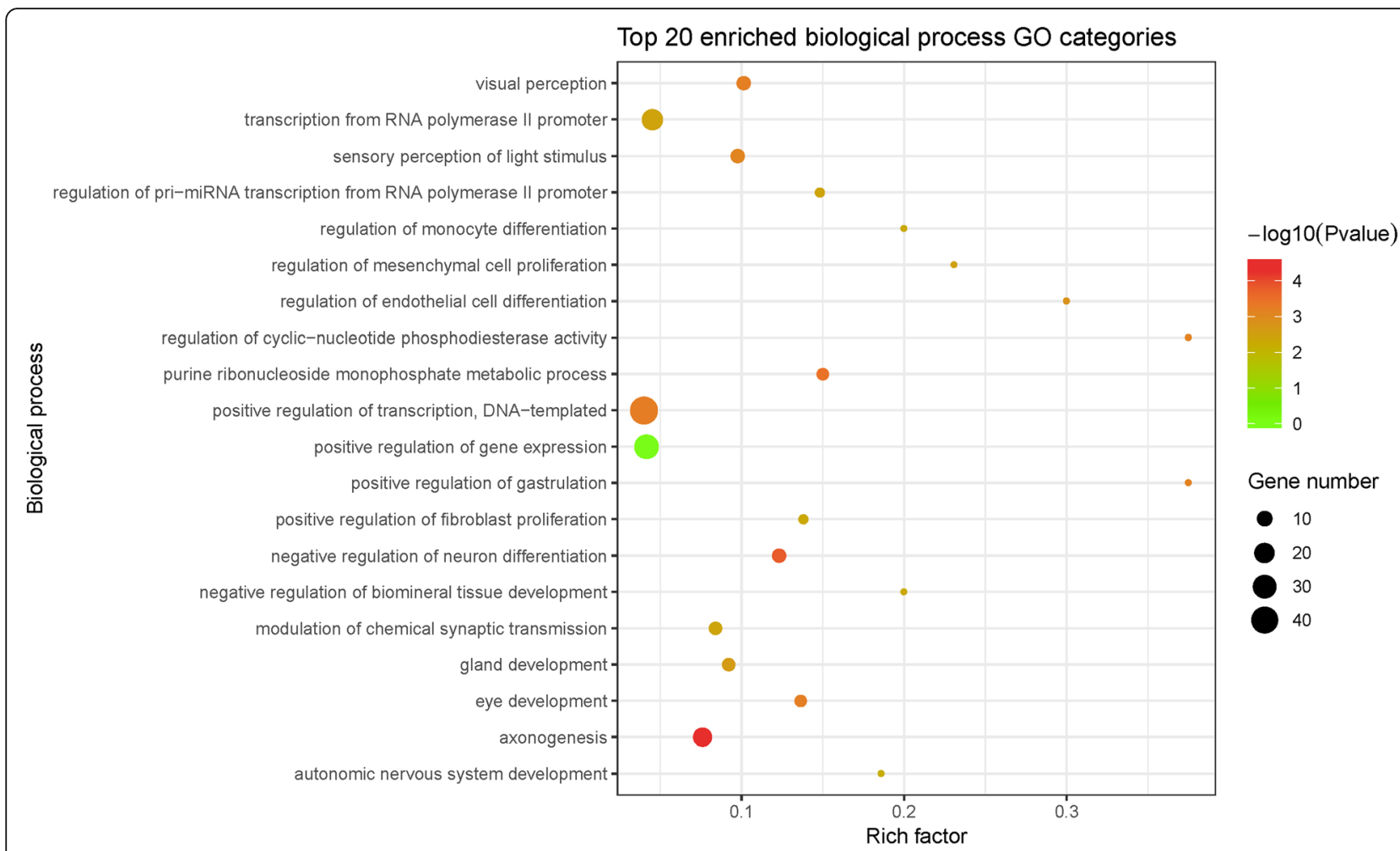

Fig. 5 GO enrichment analysis of the retinal lincRNA-nearby genes. Nearby genes were collected within $100 \mathrm{~kb}$ up- or downstream of lincRNAs. Rich factors of GO terms were calculated by (gene number in the collected set) / (gene number in the whole gene set)

in the ganglion cell layer in mouse retina that, together with the above facts, strongly indicated their RGC specific expression (Fig. 6).

Similar analysis also revealed that the expression of a total of 45 lincRNAs was altered in Pou4f2-null retinas (18 up-regulated and 27 down-regulated) and 25 in Isl1-null retinas (12 up-regulated and 13 downregulated). Among these differentially expressed lincRNAs, only three were upregulated and nine were downregulated in both Pou4f2-null and Isl1-null retinas.

\section{Discussion}

A growing number of lncRNAs have been characterized for their biological roles in diverse cellular and molecular processes, including visual maintenance and impairment [7]. Recent omics strategy based studies have greatly increased our knowledge about lncRNAs in mouse eye / retina, such as their tissue specificity in mouse eye and their expression in particular retinal neuron cell $[21,23]$. Nevertheless, so far there is still lack of a comprehensive annotation of lncRNAs expressed in all the cell types of mouse retina during its formation.

In this work, by using long-reads sequencing, we identified about 28,000 noncoding transcripts that were expressed in embryonic and neonatal mouse retinas and characterized 940 multiple-exon encoded lincRNAs. About 13\% of these lincRNAs were transcribed from previously annotated genes, as well as about $78 \%$ of them were novel isoforms. Notably, $42 \%$ of these identified lincRNAs were transcribed from the unannotated loci of photoreceptors (392/940). Therefore, apart from providing a comprehensive data set of mouse lncRNAs, this work also indicates that, maybe ascribed to the high spatio-temporal specificity of lncRNA expression and the resolution of the short reads in distinguishing the isoforms, the current annotation of mouse lncRNAs is far from complete.

According to the study on lncRNAs expressed in 15 mouse tissues (Zhao et al., 2016), lncRNA genes in each tissue are closely related to the tissue's physiological functions. By using microarray experiments, Chen et al. (2017) investigated the lncRNA genes expressed in different tissues of newborn (P0) and adult (8 week-old) mouse eyes and demonstrated the high functional correlation between the lncRNAs and the tissues. For instance, GO enrichment analysis of protein-coding genes adjacent to retina-specific IncRNAs showed that, similar to the observation of this work, several retinal cell specificity and visual function associated biological processes were 
Table 2 A list of lincRNAs differentially expressed after the functional loss of Math5, Is/1 or Pou4f2

\begin{tabular}{|c|c|c|c|c|c|}
\hline \multirow[t]{2}{*}{ Transcript } & \multirow[t]{2}{*}{ Host gene } & \multicolumn{3}{|c|}{ Expression Variation (log2Foldchange) } & \multirow{2}{*}{$\begin{array}{l}\text { Nearby } \\
\text { protein-coding } \\
\text { gene }\end{array}$} \\
\hline & & Pou4f2-null & $|s| 1-n u l \mid$ & Math5-null & \\
\hline PB.9897.1 & - & -3.6 & -3.6 & - & 1600014C23Rik \\
\hline PB.1163.1 & RP24-231D14.1 / Gm5532 & 1.6 & - & - & Acbd6 \\
\hline PB.21043.1 & - & -1.8 & -2.8 & - & Adprhl1 \\
\hline PB.15635.1 & RP23-301 M3.1 & -1.0 & - & - & Ak4 \\
\hline PB.19692.1 & - & - & -1.1 & - & Akt2 \\
\hline PB.15291.1 & - & 3.6 & - & - & Aldh1b1 \\
\hline PB.1920.1 & - & 1.5 & 1.4 & - & $\operatorname{Atg} 5$ \\
\hline PB.13742.1 & RP23-3D20.2 & -2.2 & -4.8 & -1.2 & Car2 \\
\hline PB.235.1 & - & - & - & -1.2 & Ccdc115 \\
\hline PB.3841.6 & RP24-402O2.2 & - & - & 1.5 & Ccdc182 \\
\hline PB.12472.1 & - & 2.6 & - & 2.7 & Cdca7 \\
\hline PB.19030.1 & RP23-305C 1.1 & - & 1.1 & 1.0 & Cntn4 \\
\hline PB.12202.1 & - & - & 1.8 & - & Cntrl \\
\hline PB.16460.1 & - & - & 1.3 & 1.3 & Cyp51 \\
\hline PB.16304.1 & - & 2.3 & - & - & Disp3 \\
\hline PB.2627.1 & RP24-570 M12.3 & -3.8 & -3.8 & - & Dyrk2 \\
\hline PB.7138.1 & RP24-67|21.3 & 2.1 & 2.1 & - & Entpd4 \\
\hline PB.13067.1 & RP23-401 J24.3 & - & - & -1.4 & Erv3 \\
\hline PB.23709.1 & - & - & - & -1.5 & Etd \\
\hline PB.11813.1 & E330013P04Rik & 1.2 & - & 2.4 & Fam204a \\
\hline PB.5217.1 & - & 1.1 & - & - & Foxn3 \\
\hline PB.12325.1 & - & - & -1.0 & - & Galnt13 \\
\hline PB.12831.1 & RP23-230H3.7 & -2.6 & -2.6 & - & Gjd2 \\
\hline PB.12831.2 & RP23-230H3.2 & - & 3.0 & - & Gjd2 \\
\hline PB.24411.4 & - & - & - & 1.6 & Gm21860 \\
\hline PB.421.1 & RP24-146 K19.1 & -2.9 & - & -1.5 & Hsfy2 \\
\hline PB.11544.1 & RP23-167 K23.1 & - & - & 2.1 & $\mathrm{Htr} 7$ \\
\hline PB.5127.1 & - & - & - & -2.1 & $|r f 2 b p|$ \\
\hline PB.16019.1 & - & -3.3 & -3.3 & - & Matn1 \\
\hline PB.14015.1 & RP23-403H2.2 & 2.6 & - & 2.7 & Mgarp \\
\hline PB.12783.1 & $\mathrm{RP} 23-300 \mathrm{H} 23.1$ & -1.2 & - & - & Mpped2 \\
\hline PB.13695.1 & RP24-558H21.4 & - & - & -1.2 & Mrps28 \\
\hline PB.18509.1 & RP24-83C9.4 & - & - & 1.1 & $\mathrm{Nfe} 2 \mathrm{l3}$ \\
\hline PB.22226.1 & - & - & 4.0 & - & Nrp1 \\
\hline PB.17616.4 & AC121564.2 & - & - & 1.1 & Orai1 \\
\hline PB.7358.1 & RP23-67A17.1 & - & - & -3.8 & Pou4f1 \\
\hline PB.7356.2 & RP23-67A17.1 & -3.0 & - & -3.6 & Pou4f1 \\
\hline PB.7360.1 & RP23-67A17.1 & -2.2 & - & -2.2 & Pou4f1 \\
\hline PB.21631.1 & - & - & - & -1.5 & Pou4f2 \\
\hline PB.10720.8 & - & -2.3 & - & -5.6 & Pou4f3 \\
\hline PB.10720.6 & - & -3.4 & - & -5.4 & Pou4f3 \\
\hline PB.9822.74 & - & - & - & -1.0 & Prrc2a \\
\hline PB.4404.1 & - & -1.0 & -3.6 & -2.0 & Rab10 \\
\hline
\end{tabular}


Table 2 A list of lincRNAs differentially expressed after the functional loss of Math5, Is/1 or Pou4f2 (Continued)

\begin{tabular}{|c|c|c|c|c|c|}
\hline \multirow[t]{2}{*}{ Transcript } & \multirow[t]{2}{*}{ Host gene } & \multicolumn{3}{|c|}{ Expression Variation (log2Foldchange) } & \multirow{2}{*}{$\begin{array}{l}\text { Nearby } \\
\text { protein-coding } \\
\text { gene }\end{array}$} \\
\hline & & Pou4f2-null & Is|1-null & Math5-null & \\
\hline PB.12105.1 & - & - & - & 3.8 & Rapgef1 \\
\hline PB.17233.2 & - & - & - & -1.3 & Rasgef1b \\
\hline PB.14276.3 & RP23-168E14.7 & - & - & 2.4 & Rhbg \\
\hline PB.14275.1 & RP23-168E14.7 & - & - & 6.0 & Rhbg \\
\hline PB.14276.1 & RP23-168E14.7 & - & - & 7.7 & Rhbg \\
\hline PB.5261.4 & - & 1.0 & - & - & $\operatorname{Rin} 3$ \\
\hline PB.14453.1 & AC131746.2 & -3.9 & -1.1 & -1.6 & $\sec 22 b$ \\
\hline PB.20752.2 & RP23-145H5.1 & -1.2 & - & - & Sec23ip \\
\hline PB.20752.3 & RP23-145H5.1 & -1.6 & - & - & Sec23ip \\
\hline PB.10765.1 & - & - & - & -3.3 & Sema6a \\
\hline PB.10303.1 & - & 1.2 & 1.5 & - & Six3 \\
\hline PB.13384.1 & RP23-392P11.4 & 1.1 & - & - & Slc32a1 \\
\hline PB.13385.1 & RP23-392P11.4 & 2.6 & - & - & Slc32a1 \\
\hline PB.18900.1 & - & -1.2 & - & - & Slc6a6 \\
\hline PB.11179.1 & - & - & 1.5 & - & Ssh3 \\
\hline PB.20143.3 & RP24-78 M9.4 & - & - & 1.3 & Synm \\
\hline РВ.2545.1 & - & 1.2 & - & - & Syt1 \\
\hline PB.20893.1 & AC012382.2 & 4.2 & - & - & Th \\
\hline PB.20294.2 & RP23-124O24.4 & - & - & -1.2 & Tmem126b \\
\hline PB.580.1 & - & -1.5 & - & - & Tnp1 \\
\hline PB.2344.1 & RP24-538A12.2 & -1.8 & - & -3.2 & - \\
\hline PB.11002.1 & - & 1.2 & - & -2.8 & - \\
\hline PB.5665.1 & RP23-235 J20.5 & - & - & -2.8 & - \\
\hline PB.15109.1 & - & -1.2 & - & -2.6 & - \\
\hline PB.19035.1 & - & - & - & -2.5 & - \\
\hline PB.20042.1 & Snhg14 & - & - & -2.4 & - \\
\hline PB.23395.1 & RP23-357C 16.1 & - & - & -1.5 & - \\
\hline PB.10822.2 & - & - & -1.8 & -1.4 & - \\
\hline PB.7625.1 & RP24-112|4.1 & -1.6 & - & -1.4 & - \\
\hline PB.14876.1 & RP23-473G18.1 & -1.8 & - & -1.1 & - \\
\hline PB.20300.1 & RP24-267C3.1 & - & - & -1.1 & - \\
\hline PB.6399.1 & RP23-110 M15.1 & - & - & -1.0 & - \\
\hline PB.6163.26 & RP23-88A13.2 & - & - & 1.1 & - \\
\hline PB.22575.1 & RP23-442 M18.1 & - & - & 1.2 & - \\
\hline PB.1089.1 & - & - & - & 1.5 & - \\
\hline PB.23389.1 & - & - & - & 2.0 & - \\
\hline PB.313.1 & RP23-176 M9.1 & - & - & 2.1 & - \\
\hline PB.13925.1 & - & -3.3 & - & 2.4 & - \\
\hline PB.8561.1 & RP23-127A21.3 & 5.1 & - & 4.0 & - \\
\hline PB.17246.1 & - & - & 1.4 & - & - \\
\hline PB.2422.6 & Rmst & -1.0 & - & - & - \\
\hline РВ.2474.1 & RP24-447 J10.1 & 2.2 & - & - & - \\
\hline PB.4459.2 & RP23-329 M17.1 & -4.8 & -4.8 & - & - \\
\hline
\end{tabular}


Table 2 A list of lincRNAs differentially expressed after the functional loss of Math5, Is/1 or Pou4f2 (Continued)

\begin{tabular}{|c|c|c|c|c|c|}
\hline \multirow[t]{2}{*}{ Transcript } & \multirow[t]{2}{*}{ Host gene } & \multicolumn{3}{|c|}{ Expression Variation (log2Foldchange) } & \multirow{2}{*}{$\begin{array}{l}\text { Nearby } \\
\text { protein-coding } \\
\text { gene }\end{array}$} \\
\hline & & Pou4f2-null & |s|1-null & Math5-null & \\
\hline PB.4689.1 & - & - & 1.5 & - & - \\
\hline PB.6169.1 & - & -1.5 & - & - & - \\
\hline PB.6894.35 & RP23-268P13.4 & - & -1.5 & - & - \\
\hline PB.7962.1 & - & - & 3.6 & - & - \\
\hline
\end{tabular}

overrepresented, such as retinal cone cell development, synapse assembly, axonogenesis and retina development in camera-type eye. In this work, a further investigation of IncRNA function was performed, by identifying the lincRNAs that were associated with the key RGC regulators. A total of 90 differentially expressed lincRNAs were identified after function loss of Math5, Isl1 and Pou4f2, with more than a half of them (50/90) were not detected in the photoreceptors, indicating the close relation of these lincRNAs to the cellular processes in RGCs and the cell type specificity for some of them such as $\operatorname{linc}-3 a,-3 b$ and $-3 c$, which were further supported by the FISH detection. We have also noticed that, despite the functional redundancy, Pou $4 f 2$ and Isl1 regulated a set of varied
lincRNAs, as shown previously that they regulated distinct but overlapping set of protein-coding genes [51], suggesting the roles of lincRNAs in the precise regulatory networks of retinal neuron cell differentiation and development.

Consistent with the understanding that lncRNAs are commonly lowly expressed, most lincRNAs identified in this work showed a low abundance. However, we also found high abundance for some lincRNAs with several of them were widely expressed and functional associated with retinal development, i.e., Miat, Malat1, Rncr3 and potentially Lockd, raising up the possibility that the other high expressed lincRNAs could be involved in the regulation of retinal development as well. Similarly, further studies are needed to

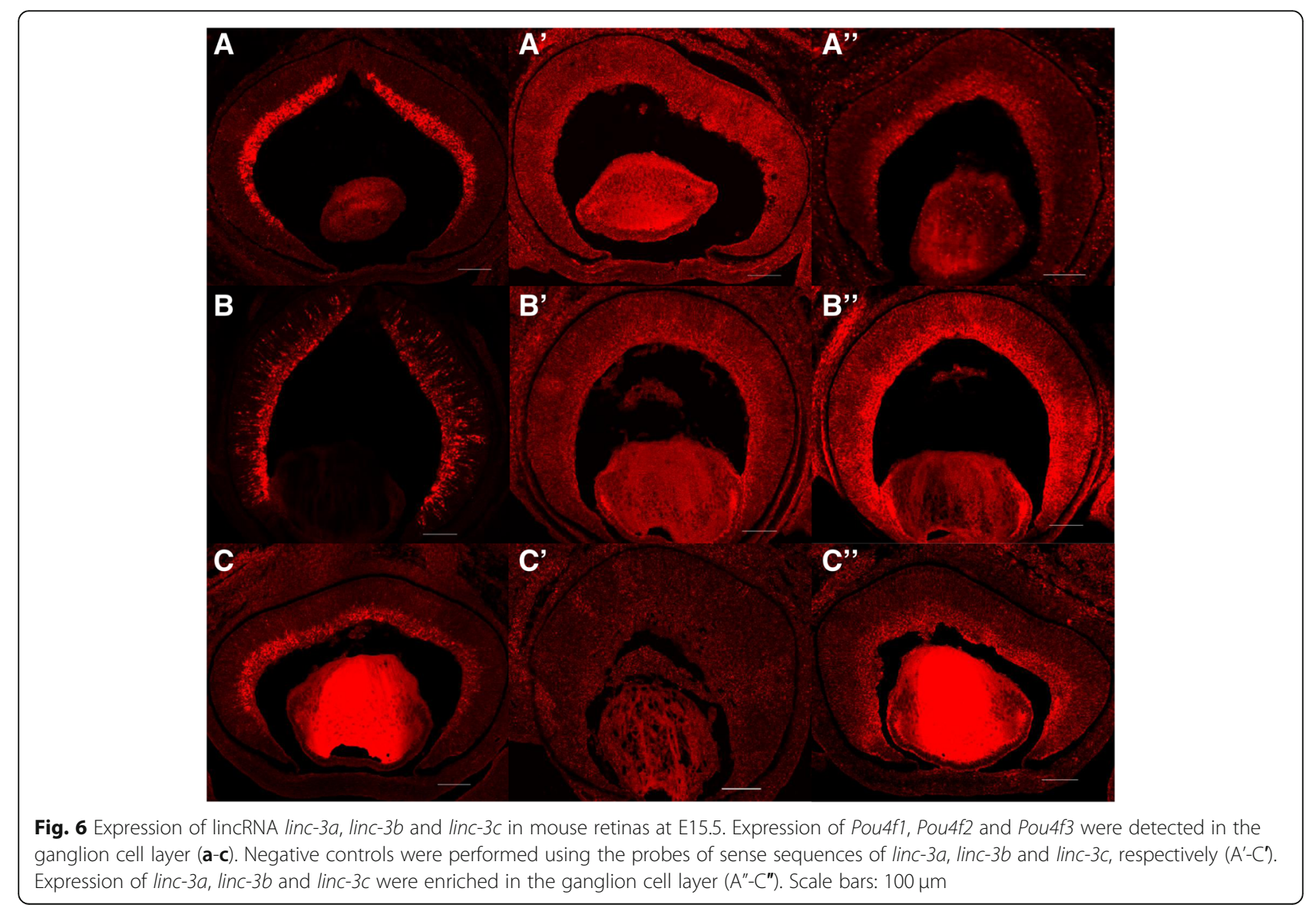


elucidate the importance in retina/eye development of many lincRNAs that are found nearby a series of key regulators of retinal cell differentiation and axon genesis.

Taking advantage of the long reads, we have identified thousands of noncoding transcripts in this work, demonstrating the high complexity of lncRNA expression in mouse retinas. Due to the fact that the DNA strand encoding these transcripts is unknown, their coding potentials were calculated for both strands, which sometimes leads to an increase of false positives for lncRNA prediction. In addition, the identified noncoding transcripts may also contain the ones overlapping protein-coding genes and potential fragments of alternative splicing forms of coding genes. It is noted that the average length of 940 retinal expressed lincRNAs is much longer than the known ones (about two-fold), but it is similar to that of the consensus sequences from iso-seq (with the average length about $2900 \mathrm{nt}$ ), indicating that the longer sequence is more likely due to the Pacbio long-reads sequencing technology, but not the strategy of lincRNA identification. However, as limited number of lincRNAs share the same structures with known ones, we could not conclude if iso-seq has generated more full-length transcripts of lincRNAs, although it is the design. It is also interesting that the number of single-exon encoded noncoding transcripts identified in this work is much more than expected. Inferred from the result of short reads mapping, i.e., at least two short reads could be aligned to $93 \%$ of the single-exon transcripts, most of the identified single-exon noncoding transcripts were expressed in the retinas. However, whether they are uncharacterized fragments of known genes or independent lncRNA genes needs to be further validated.

\section{Conclusions}

Here, we reported a systematic annotation and function analysis of lincRNAs expressed in developmental mouse retinas. Long-reads sequencing and subsequent analysis in this study identified hundreds of retinal lincRNAs, which showed the high complexity of lncRNA genes in transcription and the high specificity in retina tissue. Further analysis revealed that the function of these lincRNAs were closely related to the retinal development, such as a series of lincRNAs were associated with the key regulators of RGC differentiation (i.e., Math5, Isl1 and Pou4f2), including three lincRNAs enriched in RGCs. Summarily, this study provided a comprehensive annotation of mouse retinal lincRNAs and implied their crucial roles in the regulation of neuron cell differentiation and retinal development.

\section{Additional files}

Additional file 1: Table S1. Statistics of reads from Iso-seq before and after short reads correction. (XLSX $13 \mathrm{~kb}$ )

Additional file 2: Table S2. PCR primer pairs used for lincRNA validation. Each primer pair spans at least one intron. (XLSX $14 \mathrm{~kb}$ )

Additional file 3: Table S3. Comparison of the chromosomal location between lincRNAs identified in this work and transcripts in mouse photoreceptors. Annotation of photoreceptor transcripts is public available in NCBI (Gene Expression Omnibus, GSE74660). (XLSX 51 kb)

Additional file 4: Table S4. Functional enrichment analysis of genes nearby potential retina-specific lincRNAs. (XLSX 19 kb)

Additional file 5: Table S5. Functional enrichment analysis of genes nearby lincRNAs that are differentially expressed after function loss of Math5, Is/1 and Pou4f2. (XLSX $21 \mathrm{~kb}$ )

Additional file 6: Figure S1. Reverse transcription PCR amplification of ten randomly selected lincRNAs. Primer pairs are designed to amplify the fragments spanning at least one intron. All products (> $100 \mathrm{bp}$ ) are sequenced and analyzed. Bands representing the target region are indicated by red arrows. (TIF $3682 \mathrm{~kb}$ )

Additional file 7: Figure S2. Gene structures of linc-3a (A), linc-3c (B) and their overlapped genes. Blue boxes and lines between them indicate exons and introns, respectively. All the gene features are drawn to scale. (TIF $56 \mathrm{~kb}$ )

Additional file 8: File S1. Probes for in situ hybridization analysis of linc$3 a$, linc-3b and linc-3c. (DOC $33 \mathrm{~kb}$ )

Additional file 9: File S2. Chromosomal location of lincRNAs identified in mouse retina. Gene names are assigned when transcripts overlap known IncRNA genes (GENCODE version M17). (GTF 391 kb)

\section{Abbreviations}

CSS: circular consensus sequence; FLNC: full-length non chimera; lincRNA: intergenic long noncoding RNA; IncRNA: long noncoding RNA; nCRNA: non-coding RNA; NFL: non full-length; ORF: open reading frame; $\mathrm{RGC}$ : retinal ganglion cell; $\mathrm{RPC}$ : retinal progenitor cell

\section{Acknowledgments}

Not applicable.

\section{Authors' contributions}

L.G. and D.Y. conceived the study. Y. W1 and Y. W2 performed animal management and biosample collection. Y. W1, X. L., D. Z. and X. Z. performed molecular experiments. D.Y. and H. C. performed data analysis. D.Y., G.L. and L. G. wrote the manuscript. All these authors read and approved the final manuscript.

\section{Funding}

This work was supported by National Natural Science Foundation of China (31771436) and grants from Zhejiang Key Laboratory of Organ Development and Regeneration in Hangzhou Normal University to D.Y., The National Institute of Health grant 5R01EY026614 to L.G., and the Research to Prevent Blindness challenge grant to the Department of Ophthalmology at the University of Rochester.

\section{Availability of data and materials}

All the raw data generated in this study are available in Sequence Read Archive (SRA) of NCBI under the project PRJNA514424 (www.ncbi.nlm.nih. gov/sra/PRJNA514424). RNA-seq data of retinas from wild type, Math5-null, Is/1-null and Pou4f2-null mice at E14.5 were downloaded from NCBI Sequence Read Archive (SRA) under the accession number SRP037539.

\section{Ethics approval and consent to participate}

Mouse experiments in this study were performed according to protocols approved by the Ethics Committee for Experimental Animals at Hangzhou Normal University (permit number: 2018069). 


\section{Consent for publication}

Not applicable.

\section{Competing interests}

The authors declare that they have no competing interests.

\section{Author details}

'College of Life and Environmental Sciences, Hangzhou Normal University, Hangzhou, China. ${ }^{2}$ Zhejiang Key Laboratory of Organ Development and Regeneration, Hangzhou Normal University, Hangzhou, China. ${ }^{3}$ Zhejiang Academy of Medical Sciences, Hangzhou, China. ${ }^{4}$ Key Laboratory of microbiological technology and Bioinformatics in Zhejiang Province, Hangzhou, China. ${ }^{5}$ Department of Ophthalmology and Flaum Eye Institute, University of Rochester, Rochester, NY 14642, USA.

\section{Received: 17 January 2019 Accepted: 12 June 2019 \\ Published online: 08 July 2019}

\section{References}

1. Cepko CL, Austin CP, Yang X, Alexiades M, Ezzeddine D. Cell fate determination in the vertebrate retina. Proc Natl Acad Sci U S A. 1996;93(2): 589-95

2. Livesey FJ, Cepko CL. Vertebrate neural cell-fate determination: lessons from the retina. Nat Rev Neurosci. 2001:2(2):109-18.

3. Masland $\mathrm{RH}$. The neuronal organization of the retina. Neuron. 2012;76(2): 266-80.

4. Xiang M. Intrinsic control of mammalian retinogenesis. Cellular and molecular life sciences: CMLS. 2013;70(14):2519-32.

5. Harada T, Harada C, Parada LF. Molecular regulation of visual system development: more than meets the eye. Genes Dev. 2007;21(4):367-78.

6. Bassett EA, Wallace VA. Cell fate determination in the vertebrate retina. Trends Neurosci. 2012;35(9):565-73.

7. Wan P, Su W, Zhuo Y. Precise long non-coding RNA modulation in visual maintenance and impairment. J Med Genet. 2017:54(7):450-9.

8. Sanuki R, Onishi A, Koike C, Muramatsu R, Watanabe S, Muranishi Y, Irie S, Uneo S, Koyasu T, Matsui R, et al. miR-124a is required for hippocampal axogenesis and retinal cone survival through Lhx2 suppression. Nat Neurosci. 2011;14(9):1125-34.

9. Georgi SA, Reh TA. Dicer is required for the transition from early to late progenitor state in the developing mouse retina. J Neurosci. 2010;30(11): 4048-61.

10. Rinn $\lrcorner$, Chang HY. Genome regulation by long noncoding RNAs. Annu Rev Biochem. 2012;81:145-66.

11. Pauli A, Valen E, Lin MF, Garber M, Vastenhouw NL, Levin JZ, Fan L, Sandelin A, Rinn $J$, Regev A, et al. Systematic identification of long noncoding RNAs expressed during zebrafish embryogenesis. Genome Res. 2012;22(3):577-91.

12. Fatica A, Bozzoni I. Long non-coding RNAs: new players in cell differentiation and development. Nat Rev Genet. 2014;15(1):7-21.

13. Clark BS, Blackshaw S. Understanding the role of IncRNAs in nervous system development. Adv Exp Med Biol. 2017;1008:253-82.

14. Young TL, Matsuda T, Cepko CL. The noncoding RNA taurine upregulated gene 1 is required for differentiation of the murine retina. Current biology : CB. 2005;15(6):501-12.

15. Rapicavoli NA, Poth EM, Blackshaw S. The long noncoding RNA RNCR2 directs mouse retinal cell specification. BMC Dev Biol. 2010;10:49.

16. Rapicavoli NA, Poth EM, Zhu H, Blackshaw S. The long noncoding RNA Six3OS acts in trans to regulate retinal development by modulating Six3 activity. Neural Dev. 2011;6:32.

17. Meola N, Pizzo M, Alfano G, Surace EM, Banfi S. The long noncoding RNA Vax2os1 controls the cell cycle progression of photoreceptor progenitors in the mouse retina. Rna. 2012;18(1):111-23.

18. Yao J, Wang XQ, Li YJ, Shan K, Yang H, Wang YN, Yao MD, Liu C, Li XM, Shen $Y$, et al. Long non-coding RNA MALAT1 regulates retinal neurodegeneration through CREB signaling. EMBO molecular medicine. 2016;8(4):346-62.

19. Liu JY, Yao J, Li XM, Song YC, Wang XQ, Li YJ, Yan B, Jiang Q. Pathogenic role of IncRNA-MALAT1 in endothelial cell dysfunction in diabetes mellitus. Cell Death Dis. 2014;5:e1506.

20. Krol J, Krol I, Alvarez CP, Fiscella M, Hierlemann A, Roska B, Filipowicz W. A network comprising short and long noncoding RNAs and RNA helicase controls mouse retina architecture. Nat Commun. 2015;6:7305.
21. Zelinger L, Karakulah G, Chaitankar V, Kim JW, Yang HJ, Brooks MJ, Swaroop A. Regulation of noncoding transcriptome in developing photoreceptors by rod differentiation factor NRL. Invest Ophthalmol Vis Sci. 2017:58(11):4422-35.

22. Breschi A, Gingeras TR, Guigo R. Comparative transcriptomics in human and mouse. Nat Rev Genet. 2017;18(7):425-40.

23. Chen W, Yang S, Zhou Z, Zhao X, Zhong J, Reinach PS, Yan D. The long noncoding RNA landscape of the mouse eye. Invest Ophthalmol Vis Sci. 2017:58(14):6308-17.

24. Sondereker KB, Stabio ME, Jamil JR, Tarchick MJ, Renna JM. Where you cut matters: a dissection and analysis guide for the spatial orientation of the mouse retina from ocular landmarks. Journal of visualized experiments : JoVE. 2018:(138)

25. Salmela L, Rivals E. LoRDEC: accurate and efficient long read error correction. Bioinformatics. 2014;30(24):3506-14.

26. Wu TD, Watanabe CK. GMAP: a genomic mapping and alignment program for mRNA and EST sequences. Bioinformatics. 2005;21(9):1859-75.

27. Fu L, Niu B, Zhu Z, Wu S, Li W. CD-HIT: accelerated for clustering the nextgeneration sequencing data. Bioinformatics. 2012;28(23):3150-2.

28. Dobin A, Davis CA, Schlesinger F, Drenkow J, Zaleski C, Jha S, Batut P, Chaisson M, Gingeras TR. STAR: ultrafast universal RNA-seq aligner. Bioinformatics. 2013;29(1):15-21.

29. Wang L, Park HJ, Dasari S, Wang S, Kocher JP, Li W. CPAT: coding-potential assessment tool using an alignment-free logistic regression model. Nucleic Acids Res. 2013;41(6):e74.

30. Altschul SF, Madden TL, Schaffer AA, Zhang J, Zhang Z, Miller W, Lipman DJ, Gapped BLAST. PSI-BLAST: a new generation of protein database search programs. Nucleic Acids Res. 1997;25(17):3389-402.

31. Finn RD, Coggill P, Eberhardt RY, Eddy SR, Mistry J, Mitchell AL, Potter SC, Punta M, Qureshi M, Sangrador-Vegas A, et al. The Pfam protein families database: towards a more sustainable future. Nucleic Acids Res. 2016;44(D1): D279-85.

32. Trapnell C, Roberts A, Goff L, Pertea G, Kim D, Kelley DR, Pimentel H, Salzberg SL, Rinn JL, Pachter L. Differential gene and transcript expression analysis of RNA-seq experiments with TopHat and cufflinks. Nat Protoc. 2012;7(3):562-78.

33. Fang S, Zhang L, Guo J, Niu Y, Wu Y, Li H, Zhao L, Li X, Teng X, Sun X, et al. NONCODEV5: a comprehensive annotation database for long non-coding RNAs. Nucleic Acids Res. 2018;46(D1):D308-14.

34. Zhao Y, Liu W, Zeng J, Liu S, Tan X, Aljohi H, Hu S. Identification and analysis of mouse non-coding RNA using transcriptome data. Sci China Life Sci. 2016;59(6):589-603.

35. Langmead B, Trapnell C, Pop M, Salzberg SL. Ultrafast and memory-efficient alignment of short DNA sequences to the human genome. Genome Biol. 2009:10(3):R25.

36. Ren H, Wang G, Chen L, Jiang J, Liu L, Li N, Zhao J, Sun X, Zhou P. Genomewide analysis of long non-coding RNAs at early stage of skin pigmentation in goats (Capra hircus). BMC Genomics. 2016;17:67.

37. Bao Z, Yang Z, Huang Z, Zhou Y, Cui Q, Dong D. LncRNADisease 2.0: an updated database of long non-coding RNA-associated diseases. Nucleic Acids Res. 2019;47(D1):D1034-7.

38. Chen EY, Tan CM, Kou Y, Duan Q, Wang Z, Meirelles GV, Clark NR, Ma'ayan A. Enrichr: interactive and collaborative HTML5 gene list enrichment analysis tool. BMC bioinformatics. 2013;14:128.

39. Supek F, Bosnjak M, Skunca N, Smuc T. REVIGO summarizes and visualizes long lists of gene ontology terms. PLoS One. 2011;6(7):e21800.

40. Li R, Wu F, Ruonala R, Sapkota D, Hu Z, Mu X. Is 11 and Pou4f2 form a complex to regulate target genes in developing retinal ganglion cells. PLoS One. 2014;9(3):e92105

41. Wang L, Feng Z, Wang X, Wang X, Zhang X. DEGseq: an R package for identifying differentially expressed genes from RNA-seq data. Bioinformatics. 2010:26(1):136-8.

42. Wang SW, Kim BS, Ding K, Wang H, Sun D, Johnson RL, Klein WH, Gan L. Requirement for math 5 in the development of retinal ganglion cells. Genes Dev. 2001;15(1):24-9.

43. Trimarchi JM, Stadler MB, Roska B, Billings N, Sun B, Bartch B, Cepko CL. Molecular heterogeneity of developing retinal ganglion and amacrine cells revealed through single cell gene expression profiling. J Comp Neurol. 2007;502(6):1047-65.

44. Ogawa M, Saitoh F, Sudou N, Sato F, Fujieda H. Cell type-specific effects of p27(KIP1) loss on retinal development. Neural Dev. 2017;12(1):17. 
45. Ulitsky I, Bartel DP. lincRNAs: genomics, evolution, and mechanisms. Cell. 2013;154(1):26-46

46. Brown NL, Patel S, Brzezinski J, Glaser T. Math5 is required for retinal ganglion cell and optic nerve formation. Development. 2001;128(13): 2497-508.

47. Yang Z, Ding K, Pan L, Deng M, Gan L. Math5 determines the competence state of retinal ganglion cell progenitors. Dev Biol. 2003;264(1):240-54.

48. Gan L, Xiang M, Zhou L, Wagner DS, Klein WH, Nathans J. POU domain factor Brn-3b is required for the development of a large set of retinal ganglion cells. Proc Natl Acad Sci U S A. 1996;93(9):3920-5.

49. Gan L, Wang SW, Huang Z, Klein WH. POU domain factor Brn-3b is essential for retinal ganglion cell differentiation and survival but not for initial cell fate specification. Dev Biol. 1999;210(2):469-80.

50. Erkman L, McEvilly RJ, Luo L, Ryan AK, Hooshmand F, O'Connell SM, Keithley EM, Rapaport DH, Ryan AF, Rosenfeld MG. Role of transcription factors Brn-3. 1 and Brn-3.2 in auditory and visual system development. Nature. 1996; 381(6583):603-6.

51. Mu X, Fu X, Beremand PD, Thomas TL, Klein WH. Gene regulation logic in retinal ganglion cell development: Is|1 defines a critical branch distinct from but overlapping with Pou4f2. Proc Natl Acad Sci U S A. 2008;105(19):6942-7.

52. Jiang $Y$, Ding $Q$, Xie X, Libby RT, Lefebvre V, Gan L. Transcription factors SOX4 and SOX 11 function redundantly to regulate the development of mouse retinal ganglion cells. J Biol Chem. 2013;288(25):18429-38.

53. Xiang M, Zhou L, Macke JP, Yoshioka T, Hendry SH, Eddy RL, Shows TB, Nathans J. The Brn-3 family of POU-domain factors: primary structure, binding specificity, and expression in subsets of retinal ganglion cells and somatosensory neurons. J Neurosci. 1995;15(7 Pt 1):4762-85.

\section{Publisher's Note}

Springer Nature remains neutral with regard to jurisdictional claims in published maps and institutional affiliations.

Ready to submit your research? Choose BMC and benefit from:

- fast, convenient online submission

- thorough peer review by experienced researchers in your field

- rapid publication on acceptance

- support for research data, including large and complex data types

- gold Open Access which fosters wider collaboration and increased citations

- maximum visibility for your research: over $100 \mathrm{M}$ website views per year

At $\mathrm{BMC}$, research is always in progress.

Learn more biomedcentral.com/submissions 